\title{
lonophore-stimulation promotes re-organization of the invasion machinery of Toxoplasma gondii
}

\author{
Li-av Segev-Zarko ${ }^{1}$, Peter D. Dahlberg ${ }^{2}$, Stella Y. Sun ${ }^{3}$, Daniël M. Pelt ${ }^{4}$, James A. Sethian ${ }^{5,6}$, Wah Chiu ${ }^{1,3,7^{*}}$, John \\ C. Boothroyd ${ }^{1 *}$, \\ 1 Department of Microbiology and Immunology, Stanford University School of Medicine, Stanford, CA, USA \\ 2 Department of Chemistry, Stanford University, Stanford, CA, USA \\ 3 Department of Bioengineering, Stanford University, Stanford, CA, USA \\ 4 Leiden Institute of Advanced Computer Science, Leiden University, Leiden, The Netherlands \\ 5 Department of Mathematics, University of California, Berkeley, CA, USA \\ 6 Center for Advanced Mathematics for Energy Research Application (CAMERA), Lawrence Berkeley National \\ Laboratory, Berkeley, CA, USA \\ 7 Division of Cryo-EM and Bioimaging, SSRL, SLAC National Accelerator Laboratory, Stanford University, Menlo \\ Park, CA, USA \\ *Correspondence: wahc@stanford.edu (W.C), jboothr@stanford.edu (J.C.B)
}

\section{Abstract}

Host cell invasion by intracellular, eukaryotic parasites, like the many important species within the phylum Apicomplexa, is a remarkable and active process involving the coordinated action of many apical organelles and other structures. To date, capturing how these various structures interact during invasion has been difficult to observe in detail. Here, we used cryogenic electron tomography to generate images of the apical complex of Toxoplasma gondii tachyzoites under conditions that mimic resting parasites and those primed to invade through addition of a calcium ionophore. Using Al-based image-processing we were able to annotate 48 tomograms to identify and extract densities of the relevant subcellular organelles and accurately analyze features in 3D. We describe an interaction between an anteriorly located apical vesicle and a rhoptry tip that occurs only in the ionophorestimulated parasites and that is associated with dramatic changes in the vesicle's shape in what appears to be a stalled fusion event. We also present information to support the presumption that this vesicle originates from the well-described vesicles that parallel the intraconoidal microtubules and that the latter two structures are linked by a novel tether. Lastly, we show that a previously described rosette is found associated with more than just the anterior-most apical vesicle, indicating that multiple such vesicles are primed to enable rhoptry secretion.

\section{Introduction}

The phylum Apicomplexa includes several of the most prevalent and important human eukaryotic pathogens, such as the malaria-causing Plasmodium spp. and Toxoplasma gondii that can cause severe neurological disease in the developing fetus and those who are immunocompromised [1-3]. These obligatory intracellular parasites have a highly polarized cell shape and enter a host cell by deploying a remarkable machine at their anterior end known as the apical complex (AC), which is highly conserved and entirely specific to the 6000 species in the Apicomplexa phylum [4, 5]. Coordinate interaction of subcellular organelles and distinct elements of the cytoskeleton that comprise this extraordinary and dynamic machine allows the active invasion of these singlecelled eukaryotes into other (host) eukaryotic cells [6].

Active invasion by tachyzoites, the rapidly growing life stage of Toxoplasma, involves protrusion of a unique spiral of tubulin-based fibrils known as the conoid, a structure found only in the coccidian subgroup of apicomplexans $[5,7,8]$. During protrusion the conoid, as well as two preconoidal rings at its anterior end, move outward, away from the cell body. When protruded, these structures are anterior to the apical polar ring (APR), which 
functions as the anchoring site for the subpellicular microtubules (SPMTs) [9]. The SPMTs are arranged in a gently spiraling pattern and extend $2 / 3$ the length of the parasite to provide mechanical support [10].

During the initial stages of invasion, two distinct, apically located secretory organelles, the capsule-shaped micronemes and the club-shaped rhoptries, secrete their contents [6]. Micronemes deposit their contents onto the surface of the parasites and these proteins facilitate surface attachment of the parasite to the host cell surface [11]. Next, rhoptry proteins are injected by an unknown mechanism from the rhoptry neck into the host cytoplasm [12]. This cargo includes RON2 which then somehow integrates into the host plasma membrane and binds to a micronemal protein, AMA1, on the parasite surface. The result is a ring of tight interactions that migrates down the parasite as it invades into the host cell [13]. Following secretion from the rhoptry necks, the rhoptry bulbs inject their contents into the host cell, including effector proteins that enable the parasites to grow within and co-opt the host cell. Two additional components of the AC that are poorly understood but are likely of prime importance, are a series of apical vesicles (AVs) and a pair of intra-conoid microtubules (IMTs), along which most of the AVs are aligned, and all within the conoid space. Previous structural studies have revealed that the most anterior AV sits between the tip of one or two rhoptries and the parasite's apical plasma membrane [14-18]. This AV has been suggested to be a part of the docking machinery for rhoptries [14, 15, 19], through interactions with the plasma membrane-bound rhoptry secretory apparatus (RSA). Despite these recent advances, the exact means by which rhoptry secretion occurs and specifically the precise role of the AVs in this process, remain unknown.

Cryogenic electron tomography (cryo-ET) is a powerful tool to study cellular architecture in situ without using chemical fixatives or metal stains [20]. This method can visualize membranes and protein-based cellular structures in a near-native, frozen, hydrated state but it can be severely limited by the thickness and the inherently low contrast of biological samples. Recently, cryo-ET has proven successful in revealing the 3D organization of the subcellular organelles in the AC of Toxoplasma [8, 15, 19]; however, due to the transient nature of host cell invasion and the limited throughput of cryo-ET, it has not yet been possible to use this method to capture the invasion process itself. As an alternative to observing parasites in direct interaction with host cells, one can observe the biological machinery of extracellular parasites under internal calcium flux via stimulation with calcium ionophore that, among other things, primes the parasites for invasion by initiating protrusion of the conoid and microneme secretion. We report here the results from such an analysis including the finding that stimulation with the calcium ionophore is associated with a distortion and apparent fusion of an AV with a rhoptry tip. We also describe a neural net approach for annotation that allows rapid and precise quantitation of this and other subcellular phenomena.

\section{Results}

Following stimulation with the calcium ionophore, A23187, the conoid of Toxoplasma gondii tachyzoites harvested from infected monolayers protrudes in an irreversible way [21] and micronemal proteins such as MIC2 are deposited onto the surface of the parasites where they can be cleaved by proteases [22]. If harvested into Endo buffer containing high potassium levels, intracellular calcium levels remain stable and invasion is stalled [23, 24]. Using a phase microscope, we assessed the percentage of extracellular parasites that had undergone conoid protrusion under "stimulating" (1 $\mu \mathrm{M}$ A23187 in Hanks Balanced Salt Solution (HBSS)) and "non-stimulating" conditions (Endo buffer). The results (S1A Fig) showed $80 \%$ of the tachyzoites had protruded conoids after $0.5-2$ minutes of ionophore-stimulation while only $8 \%$ had protruded conoids even after 10 minutes incubation in nonstimulating conditions. To assess microneme secretion, a western blot was used to detect secreted MIC2 (S1B Fig). An already cleaved form of MIC2 was detected after 2 minutes of ionophore-stimulation at room temperature, while no cleaved MIC2 was detected when parasites were incubated in non-stimulating conditions. We, therefore, harvested the tachyzoites into either Endo buffer or HBSS supplemented with $1 \mu \mathrm{M}$ A23187 for $0.5-2$ minutes prior to transfer to a lacey carbon grid and plunge-freezing followed by imaging using cryo-ET. A total of 40 tomograms of stimulated and 8 tomograms of unstimulated cells were generated in this way and a representative tomogram from each condition is presented in S1 Movie and S2 Movie, respectively. Virtual sections from these tomograms are presented in Fig 1 together with a model of known components of the AC.

In all the tomograms, micronemes and rhoptries were clearly visible inside the space delimited by the conoid spiral. Microneme and rhoptry secretion are essential for successful invasion of apicomplexan parasites. Although it is hypothesized that both secretion events require a membrane fusion, no such interaction or fusion 
of the micronemes with the parasite membrane has been reported or was observed here. With regard to rhoptries, Toxoplasma carries up to a dozen such organelles, but only two rhoptry necks were observed within the intraconoid space of non-stimulated parasites. In stimulated parasites, however, the number of rhoptries that occupy the intraconoid space varied with 23 such cells displaying two rhoptries in the conoid space, 12 cells displaying only one rhoptry, and 5 that did not display any rhoptries in this space $(58 \%, 30 \%$ and $12 \%$, respectively, $\mathrm{N}=40$ ). A recent cryo-ET study of Toxoplasma tachyzoites harvested in a buffer that was not specifically reported as inducing or inhibiting conoid protrusion, described a constriction in the rhoptry neck, a phenotype that could only be detected by reconstructing the 3D volume of the rhoptries [19]. In tomograms where the rhoptries were visible, we observed a similar constriction in the rhoptry neck in a majority of the stimulated parasites (23 of 36 cells) and also in 1 of 7 non-stimulated (S2A Fig, and S3 Movie).

In addition to the microneme and rhoptries, two IMTs and a series of up to 6 AVs were also seen in the intraconoid space. Relative to the plane of the grid, the IMTs were positioned in the center of the conoid in $13 \%$ of the cells; otherwise, these IMTs were positioned to one or other side of the intraconoid space $(87 \%, N=47)$, as viewed from above. Intriguingly, there was a strong relationship between the position of the AVs relative to the IMTs and the position of the IMTs in the conoid space, such that the vesicles were almost always (31/33) found on the side of the IMT closest to the conoid fibrils $(\varphi=0.87$, Pearson correlation for two binary variables). When two rhoptries were found within the conoid space, they were always positioned on opposite sides of the IMTs, whereas when only one rhoptry was present ( $N=13$ ), it was usually (10 of 12 parasites) on the side not occupied by the AVs ( $\varphi=-0.63$, Pearson correlation for two binary variables). Tomograms of non-stimulated parasites revealed a single AV positioned at the apex of the cell, anterior to the IMTs, while tomograms of stimulated parasites revealed one, two, or even three AVs in this location (37, 3, and 1 such cells, respectively) (S2B/C Fig). Of greatest interest, however, was that in many of the ionophore-stimulated parasites there was an intimate association between one or two rhoptries and the most anterior $\mathrm{AV}$, including an apparent deformation of the $\mathrm{AV}$ membrane towards the rhoptry tip (1C/E and S2D/E Fig). This point is further explored below.

\section{A mixed-scale dense convolutional neural network allows fast and accurate annotation of distinct segments using a limited amount of training data}

One of the challenges of using cryo-ET data for studying biological phenomena is the amount of time needed to annotate and then analyze the tomograms. Obtaining large amounts of data and being able to quantify those data is especially important when observing differences between two sample conditions that might be relatively subtle and therefore prone to observer biases. To meet this challenge, we adopted and customized the MixedScale Dense (MSD) Neural Networks approach [25] to perform image segmentation and classification. Unlike traditional neural networks, MSD networks are specifically designed for scientific imaging data where there is limited amount of ground-truth training data. These networks operate by removing the upscaling and downscale in typical networks and replacing these operations by mathematical stencil operators which perform the convolutions across multiple scales ("mixed scale") without requiring generation of intermediate objects that can only communicate with nearby layers. Consequently, all layers can be connected together, hence the idea of a "dense" network.

Here, we adopt these MSD networks to quantify the effect of ionophore-stimulation on the organization of the $A V s$ within the AC. In our application, the initial training data included manual annotation of only 3-4 successive slices that contained apical vesicles from each of 7 reconstructed tomograms. IMTs are very challenging to train on because only two are present per sample and they are only visible in a few slices, and the same is true for rhoptries that are similar both in width and density to micronemes but differ in length that is hard to assess when training data are two-dimensional (2-D).

Within these 3-4 slices we manually annotated the micronemes, rhoptries, microtubules (SPMTs and IMTs), and AVs with both micronemes and rhoptries given one label, and the SPMTs and IMTs another. Combining related structures into a single label reduced errors in the network output and in most cases the difference between the two combined entities could be readily discerned in the NN output (e.g., the location of the SPMTs and IMTs are completely different, as is the shape and extent of the rhoptries and micronemes when viewed in three dimensions, features that the $\mathrm{NN}$ did not pick up on as readily as their other differences from the other structures present) (S3A/B Fig).

Rather than apply this initially trained network to all of the acquired tomograms, we used an iterative bootstrap procedure: we apply the network to a few slices, and then manually fine-tune the results to expand the 
training set. Making these corrections was much faster than manual annotation de novo and allowed for 43 additional annotated slices (3-6 slices each from 11 tomograms) to be used for training, which led to a considerable increase in accuracy.

To characterize and assess the $\mathrm{NN}$ derived annotations relative to ones made by expert human annotators, we compared the level of agreement over two parameters, pixel-wise error and connected-component error. The percentage agreement between two expert human annotators for the AVs and the micronemes/rhoptries was between $80-100 \%$ for the two parameters, respectively; this level of agreement was similar to that between each of the human experts and the NN (S3C Fig). For the microtubule's annotation, the pixel wise agreement was $70 \%$ for the two human annotators, making this the structure for which the human annotators had the least agreement, probably due to the short subpellicular microtubules seen in cross-section. This could make it challenging for the NN to learn as reflected in the $50 \%$ agreement between the human and NN annotations, although due to the large standard deviation, the difference between this and the $70 \%$ agreement for the two human annotators was not statistically significant. This iterative training based on manual correction improved the accuracy of the $\mathrm{NN}$ in annotating prominent structures, as well as in detecting low-abundance features (e.g., AVs; S4 Fig), yielding a highly accurate annotation for tomograms that were not used in the training data (Fig2). Thus, the NN process enables faster and more objective analyses of the data, including characterization of subcellular components and their possible interaction under stimulating and non-stimulating conditions, as described further below.

\section{Exposure to a calcium ionophore promotes an intimate interaction between the most ante- rior AVs and rhoptry tips}

To quantify the precise changes induced by calcium ionophore, we used the NN to analyze multiple tomograms of stimulated and non-stimulated extracellular parasites. These changes led us to explore the possibility that stimulation is affecting the position of the anterior vesicles relative to the IMTs by measuring the distance between these two structures. The results showed similar distances between the AV center-of-mass (COM) and the IMT for stimulated (median=79.3 nm, mean $\pm S D=79.3 \pm 14.6 \mathrm{~nm}, \mathrm{~N}=31$ ) and non-stimulated (median=73 nm, mean $\pm \mathrm{SD}=72 \pm 15 \mathrm{~nm}, \mathrm{~N}=8$ ) parasites (Fig 3C). We also measured the distances between the AVs' boundaries (membrane) and the IMT (Fig 3D), again revealing a similar distribution of distances (median=53.5 nm, mean \pm $\mathrm{SD}=53.3 \pm 13.7 \mathrm{~nm}, \mathrm{~N}=31$ for stimulated parasites and median=48.4 nm, mean $\pm \mathrm{SD}=47.1 \pm 19 \mathrm{~nm}, \mathrm{~N}=8$ for nonstimulated parasites), meaning the distance is not affected by the vesicle's structural characteristics. Lastly, we measured the angle between the AV and a best fit line through the nearest IMT and observed that this did not change upon stimulation, as presented in figure $3 \mathrm{E}$ (median $=18^{\circ}$, mean $\pm \mathrm{SD}=19.6 \pm 11.7^{\circ}, \mathrm{N}=31$ for stimulated parasites and median $=15.6^{\circ}$, mean $\pm \mathrm{SD}=18.8 \pm 15.4^{\circ}, \mathrm{N}=8$ for non- stimulated parasites).

One of the most striking changes we observed upon ionophore-stimulation was the deformation of the anteriormost $\mathrm{AV}$ in the form of a protrusion directed toward the tip of a nearby rhoptry. We observed this deformation in $29(81 \%)$ of the stimulated parasites $(\mathrm{N}=36)$, whereas it was not observed in any unstimulated parasites $(\mathrm{z}=4.162$, 99.9\% for Two-Sample Z-test for proportions). This deformation could be quantified using the output of the NN annotation to determine the membrane curvature of each vesicle. This curvature was assessed in the 2-D virtual slices that were within $8 \mathrm{~nm}$ of each vesicle's center of mass and was averaged as a function of angle around the vesicle ( $\mathrm{S} 5 \mathrm{Fig}$ ). The results were plotted in 10 degree increments, starting at the voxel of the vesicle that is closest to a voxel of the interacting rhoptry (Fig 4A/B). In stimulated parasites, this analysis showed a clear transition from high to low curvature when moving away from the point of interaction (i.e., a teardrop-like shape). This effect was absent in non-stimulated parasites where the curvature was relatively constant, reflecting their near spherical shape and similar to the shape of the more posterior, IMT-aligned AVs where a similar quantification was performed but the values were aligned relative to the vesicle voxel that was closest to an IMT voxel.

The teardrop shape of the rhoptry-interacting $A V$ in stimulated parasites suggests that this $A V$ is either about to fuse with the rhoptry or was recently created by budding from the most apical portion of a rhoptry. To further help distinguish between these two possibilities, we examined the region where the two structures are closely apposed in greater detail. The results showed an apparent continuity between the lumens of the rhoptry and interacting $A V$ in 12 out of 36 tomograms of ionophore-stimulated parasites (representative images are shown in Figures $1 \mathrm{~B}$ and $3 \mathrm{~A}$ ). To distinguish between fusion and fission, we compared the density of the AV's contents compared to the rhoptries; fission might predict that they should have about the same contents (since the interacting AV would be very recently derived from rhoptries), whereas if they are of completely different origins and are just initiating fusion, they might have very different cargo, as reflected in the density of their contents in the 
tomograms. The results (Fig 4C) showed that the median density of the AVs was similar, regardless of whether they were interacting with the rhoptry or one of the more posteriorly located, IMT-associated vesicles, and that this density was substantially less than the rhoptries. These results suggest, but certainly do not prove, that the $\mathrm{AVs}$ have a distinct cellular origin from rhoptries and that the interacting $\mathrm{AV}$ is in the process of fusion with, rather than budding from, the rhoptry tip. We also compared the volumes of the AVs. The results (Fig 4D) showed that the volumes were similar between the AVs, regardless of their position or whether they are stimulated. This does not distinguish between the fusion and fission hypotheses but does further indicate that the two structures are distinct and that their intimate interaction is a stable state.

\section{The AVs associate with the IMT and with each other}

In addition to the AV positioned anterior to the IMT, we observed up to 6 AVs aligned along the IMTs in the intraconoid space, with a mode of 3, regardless of whether they had been exposed to calcium ionophore (Fig $5 A$ ). These AVs were usually lined up with a regular spacing between them and a uniform distance from the IMTs, suggesting an association between these various components (Fig 5B). In 7 tomograms (5 stimulated and 2 nonstimulated parasites), we also identified apparent AVs posterior to the IMTs (S6A Fig).

Closer inspection of the IMT-aligned AVs revealed a density partially surrounding the vesicle in the majority (75\%) of the tomograms, with a similar density also observed surrounding the AVs positioned anterior to the IMTs (S7A-D Fig). Using the tomographic data to examine this structure more carefully revealed that in at least 31 out of 48 tomograms the density partially coats the AVs, and in 10 instances it resembled the rosette that was previously characterized as part of the RSA found between the rhoptry-interacting AV and the parasite's plasma membrane [19] (S8 A-D Fig). The difference in our data from those previously reported is that we observe these rosette-like structures associated with more than one $A V$ in a single $A C$ and even around AVs that are IMTassociated, not just those that are anterior-most or interacting with a rhoptry tip.

The fact that the AVs are generally aligned on just one side of the IMT pair raises the question of how the two IMTs might differ from each other. Our results showed that within any given tomogram, regardless of whether the parasites had been exposed to calcium ionophore or not, the two IMTs were similar in length (S6B Fig). We also observed an internal striation density within the lumen of both IMTs, but no external densities regularly coating one IMT or another, leaving unanswered what determines the side of the IMT pair with which the AVs associate.

Next, to determine if ionophore stimulation altered the IMT pair overall, we examined the stimulated and non-stimulated parasites and found that both the length $(350 \pm 60 \mathrm{~nm}$ for stimulated and $300 \pm 30 \mathrm{~nm}$ for nonstimulated tachyzoites) and curvature ( $0.7 \times 10-4 \mathrm{~nm}-1$ for stimulated and $1 \times 10-4 \mathrm{~nm}-1$ for non-stimulated tachyzoites) did not substantially change upon stimulation (S6B/C Fig).

To determine if ionophore-stimulation caused other changes in the AV/IMT interaction, we measured the median distance between each AV's center of mass (COM) and the nearest IMT. The results (Fig 5D) showed no change upon stimulation (median= $29.2 \mathrm{~nm}$, mean $\pm \mathrm{SD}=31.7 \pm 10.8 \mathrm{~nm}, \mathrm{~N}=119$ for stimulated parasites and median= $28.4 \mathrm{~nm}$, mean $\pm \mathrm{SD}=32.1 \pm 15.6 \mathrm{~nm}, \mathrm{~N}=23$ for non-stimulated parasites). Similarly, the distance measured between the AVs' boundaries and the IMTs showed a tight distribution of lengths that did not change upon stimulation (Fig 5E). Given their approximately spherical shape, this agreement between the results when measuring from the COM and outer edge of the AVs also indicates that the AVs are of similar size in the two conditions. To explore possible interactions that can maintain the regular distance between the vesicles and an IMT, we extracted the densities in the interface between the two structures and aligned them according to the minimal distance vector between the AV's boundary and the IMT. A subtomogram average created from 5 or 6 vesicles revealed a $25 \mathrm{~nm}$ long density apparently connecting the vesicle and the nearest IMT (Fig 4F). We also measured the distance between the COM of neighboring vesicles to determine if ionophore-stimulation caused changes in the spacing between them. The results (Fig $5 \mathrm{G}$ ) showed no change upon stimulation (median= $78.4 \mathrm{~nm}$, mean \pm $\mathrm{SD}=84.1 \pm 19.3 \mathrm{~nm}, \mathrm{~N}=84$ for stimulated parasites and median $=79.4 \mathrm{~nm}$, mean $\pm \mathrm{SD}=85 \pm 12.6 \mathrm{~nm}, \mathrm{~N}=15$ for nonstimulated parasite). Similarly, the distance between the AVs' boundaries do not change upon stimulation (Fig $5 \mathrm{H}$ ). To determine if the distribution of the AVs along the IMT change upon ionophore stimulation, we measured the distance between the most anterior IMT-associated AV to the tip of the IMT. The results showed no change upon stimulation with a mean of $40 \pm 10 \mathrm{~nm}$ for both stimulating and non-stimulating conditions (S6E Fig). Interestingly, we frequently detected 1-3 densities apparently connecting neighboring vesicles, but we were not able to average the densities due to structural heterogeneity (Fig $5 \mathrm{I}$ ). The average length of the intervesicular connections, as determined by manual annotation of 43 filaments, was $28 \pm 5 \mathrm{~nm}$ while the average distance between 
the AVs at their closest points was $22 \pm 4 \mathrm{~nm}$. Vesicles that did not have any detectable density connecting them appeared more distant from each other with average distances of $45 \pm 11 \mathrm{~nm}$ (S6F/G Fig). These data suggest the existence of distinct structures that both tether the AVs to the IMTs and that maintain a constant distance between IMT-associating AVs.

\section{Discussion}

Tomography enables determination of the full volume of objects and thereby reveals features that otherwise might be missed in analysis of electron micrographs using thin sections. Cryo-ET also benefits from involving no chemical fixation, only flash-freezing of samples. To draw reliable conclusions, however, many samples must be analyzed, and careful annotation of the tomograms is necessary to quantify observed features. The Mixed-Scale Dense Neural Net described here allowed us to train on remarkably little manually annotated data, obtaining a robust, automated workflow for image-segmentation-and-recognition across a large number of results. We were able to obtain accurate annotations from slices that were not part of the training set, and these were generally as similar to those of two expert annotators as those two annotators were to each other. We were also able to overcome the challenge presented by differences in the signal-to-noise ratios in different tomograms as the NN successfully annotated tomograms that were not part of the training set. As a result, we were able to quickly and accurately annotate and then quantify features in a large number of tomograms. Subtomogram-averaging of the features thereby detected allowed us to discover structures not previously reported (e.g., densities connecting AVs to the IMTs), as well as characterize differences produced by ionophore stimulation (e.g., direct interconnection of the lumens of the anterior-most AV and a rhoptry tip). Using corrected NN annotations as additional training data to improve the NN's accuracy is an important advance over existing pipelines as it reduces the manual annotation time dramatically.

Inspection of the tomograms revealed that the plasma membrane was clearly visible and intact in less than half the tomograms under both conditions. We note that tomograms presenting impaired plasma membrane (likely due to the freeze plunge step to remove excess liquid by blotting) could have added noise to the relative positions of the subcellular organelles, but we observed no consistent difference between tomograms of parasites with and without visibly intact plasma membranes. Micronemes were always seen occupying the conoid space suggesting that secretion takes place at the tip of the parasite, beyond the conoid, as the region where the plasma membrane can be most easily accessed by such a large structure [22]. Microneme secretion was previously shown to involve ferlin 1 (TgFER1) and TgDOC2, both C2-domain-containing proteins [26, 27]. C2-domains conditionally engage with lipids in a calcium-dependent manner, facilitating membrane fusion [28]. It has therefore been suggested that micronemes directly fuse with the parasite membrane to discharge their contents [11, 29]. The calcium ionophore used here was previously shown to induce microneme secretion at $37^{\circ} \mathrm{C}$ [22]. Here, we used cooler, room-temperature conditions and still observed such a release. Although we hoped to possibly capture active microneme secretion in our tomograms, we did not identify any apparent fusion between these organelles and the plasma membrane. Part of the reason for this could be that the plasma membrane was clearly visible and generally intact in only $52 \%$ of the tomograms under both conditions (25 out of 48 tomograms). It may also be that the fusion process is very short-lived and therefore difficult to capture.

Rhoptry secretion has been detected only upon contact with a host cell, but the exact nature of the signal required and the process leading to such secretion are unclear. Docking of rhoptries to an AV and RSA within the conoid prior to egress could potentially enable a very rapid response once contact with a host cell has been made. Constriction of the rhoptry neck was recently reported in Toxoplasma tachyzoites, in areas of the neck that are devoid of luminal filaments [19] and our results indicate that this phenomenon is not stimulation-dependent. The purpose of these constrictions and whether the arrangements of the luminal filaments are dynamic remains to be determined.

The role of the AVs in rhoptry secretion has long been speculated [14,30] and they were previously described in other apicomplexans, e.g. Cryptosporidium, Eimeria, Sarcocystis, and Besnoitia [15-17, 19, 31]. Although no direct connection was detected between the IMTs or their associated AVs and the conoid fibrils, we did observe that the AVs are not centered or positioned randomly in the intraconoid space; instead, most were positioned to the side, as viewed from the top of the specimen. Although compression of the cells (due to blotting) can potentially affect the positioning of features within the intraconoidal space, the side positioning of the AVs and IMTs was observed also in cells that were less compressed. Therefore, we speculate that the asymmetric organization 
of features within the intraconoidal space might reflect the shape of the tachyzoite cell; i.e., the crescent-shape of the parasite could influence how it is lying on the surface of the grid during sample preparation. Unfortunately, our limited field of view did not allow us to observe the entire tachyzoite or its overall curve, but it seems most likely that they would rest on one or other side since lying with the curve facing up or down would be much less stable. Furthermore, we observed that the majority of the IMTs were not centered within the intraconoid space, and the aligned vesicles were on the side of the IMTs closest to the conoid fibrils. We did not find any structural difference between the two IMTs that might explain which IMT interacts with the AVs. What governs the organization of these vesicles next to one IMT remains to be determined, although we did note the presence of an apparent density or tether that was reproducibly present between the AVs and IMT, as well as between AVs aligned along this structure.

It was recently suggested that an AV facilitates the docking of a rhoptry to the parasite membrane through a newly revealed entity named the rhoptry secretory apparatus (RSA), and that the AV-rosette connection is independent of rhoptry docking [19]. We extend those results by reporting that the rosette structure that was previously seen within the RSA of the anterior-most AV is also apparent next to other AVs, either associated or not with the IMTs. We also observed that upon treatment with a calcium-ionophore, a known stimulator of invasion, a distended $\mathrm{AV}$ was seen fused with a rhoptry tip, and this connection appears to be stable enough to be captured in most such tomograms. This result is in agreement with another observation that links calcium signaling to rhoptry secretion, where depletion of the calcium-sensitive ferlin 2 (TgFER2) directly blocks rhoptry secretion [32]. Moreover, mass spectrometry analysis identified TgFER2 as an interacting partner of Nd9 together with Nd6, both part of a conserved complex essential for rhoptry secretion. Nd6 was localized to the apical tip, presumably where the anterior AV is located [15]. Whether TgFER2 facilitates the AV-rhoptry fusion remains to be determined.

The difference between the density measured for the AVs and the rhoptry favors a model where these two entities have distinct origins, rather than AVs being derived by fission from rhoptries, unless a mechanism exists to pass into the AV only a subset of the rhoptry contents. It is possible that the AV/rhoptry interaction stabilizes the position of the latter in the intraconoid space, keeping it docked through the RSA to the plasma membrane as the conoid protrudes and retracts and micronemes enter the conoid space on their way to secrete. Assuming that rhoptry proteins are indeed transported to the host cell through the RSA, it is also possible that the interaction reflects a given rhoptry's commitment towards secretion. It is unclear if more than one rhoptry secretes its contents during invasion of one host cell. Interestingly, although unstimulated parasites invariably displayed two rhoptries within the intraconoid space, upon stimulation, we mostly observed interaction between an AV and only one rhoptry tip, even when two rhoptries were in close proximity. The apparent fusion is similar to what was reported for Cryptosporidium sporozoites, where a continuity between the AV and the single rhoptry was also observed [19].

The size of the AVs and the average distance between neighboring ones predicts that a single IMT could interact with up to 6 vesicles, as we also observed, although we observed a mode of just 3 . It is unclear if there is recruitment and transport of additional AVs to the IMTs but the appearance of vesicles with all the properties of an $\mathrm{AV}$ posterior to and not associating with the IMTs suggests this is possible. Whether there is, in fact, a dynamic equilibrium of AVs will require identification of their cargo and/or other markers that allow them to be specifically labelled.

The similarity between the IMT-aligned and rhoptry-associating AVs in volume, and density, and the appearance of more than one anterior, non-IMT-associating AV in the AC of stimulated parasites supports the previous suggestion [14, 19] that all AVs have a common origin. Toxoplasma tachyzoites are capable of multiple invasion attempts where the rhoptries secrete their content into a host cell without completing invasion [33, 34]. To accomplish this, it could be that an AV stored along the IMT relocates to the apex of the cell where it can interact with a newly extended rhoptry and support their secretion after a failed invasion attempt involving a previous AV-rhoptry interaction. The rosette structure we identified associating with more than just the anterior-most AV suggests that at least part of the RSA is transported to the apex of the parasite together with a given vesicle.

Transport of vesicles along the IMTs might be mediated in part by motor proteins similar in size to the densities observed between the vesicles and the IMT, such as dynein or kinesin. However, the distance between the IMT to the anterior $\mathrm{AV}$ is greater than its distance to the IMT-associated AVs, meaning any apparent connection between them should be altered in order to allow an IMT-associated AV to reposition as a new anterior AV. Ultimately, to uncover the exact means by which the AV supports rhoptry secretion, the structure and organization of the entire apical complex will need to be examined in parasites captured in the act of invasion of host cell, a technical 
challenge but within the scope of emerging technologies with cryo-ET after focused ion-beam milling [35], superresolved cryogenic correlative light, and electron microscopy [36] and high throughput tomogram data collection and processing.

\section{Materials and methods}

\section{Parasite maintenance and cell culture}

Toxoplasma gondii RH $\Delta$ hxgprt strain was maintained by growth in confluent primary human foreskin fibroblasts (HFFs) in Dulbecco's modified Eagle's medium (DMEM; Invitrogen, Carlsbad, CA) with 10\% fetal bovine serum (FBS; HyClone, Logan, UT), $2 \mathrm{mM}$ glutamine, $100 \mathrm{U} / \mathrm{ml}$ penicillin, and $100 \mu \mathrm{g} / \mathrm{ml}$ streptomycin (cDMEM) at $37^{\circ} \mathrm{C}$ in 5\% CO2. The HFFs are fully deidentified and therefore do not constitute human subjects research.

\section{Parasite preparation}

Tachyzoites were released from heavily infected monolayers of HFFs by mechanical disruption of the monolayers using disposable scrapers and passage through a 25-gauge syringe. The parasites were added to fresh monolayers of HFFs, and 18-20 hours post-infection, were washed two times with Endo buffer (EB) (45 mM potassium sulfate, $106 \mathrm{mM}$ sucrose, $10 \mathrm{mM}$ magnesium sulfate, $20 \mathrm{mM}$ Tris buffer $\mathrm{pH} 7.2,5 \mathrm{mM}$ glucose and $0.35 \%$ bovine serum albumin). The HFFs monolayers were scraped and passage through a 27-gauge syringe and tachyzoites were released into fresh EB at room temperature. Tachyzoites were pelleted and resuspended in fresh EB.

To stimulate the parasites, tachyzoites were washed two times with cold Hank's balanced salt solution (HBSS)

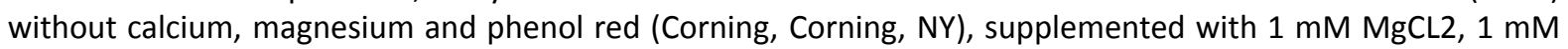
$\mathrm{CaCl} 2,10 \mathrm{mM}$ NaHCO3 and $20 \mathrm{mM}$ HEPES, pH 7. The HFFs monolayers were scraped and passage through a 27-gauge syringe and tachyzoites were released into fresh cold HBSS. Tachyzoites were pelleted and resuspended in fresh cold HBSS. Note that all steps were done on ice. Calcium-ionophore (A23187, Sigma, St. Louis, MO) at a final concentration of $1 \mu \mathrm{M}$ was added to the sample at room temperature followed by chemical fixation for conoid protrusion assay or plunge freezing of unfixed sample for cryogenic electron tomography.

\section{Conoid protrusion}

Tachyzoites were prepared as described above and treated for 0.5-2 minutes with A23187 or incubated for 10 minutes in EB at room temperature. Tachyzoites were then fixed for 20 minutes with Formaldehyde and concentrated by centrifugation. The pellet was resuspended in $30 \mu \mathrm{l}$ PBS and left to dry on a glass coverslip. Protruded and non-protruded parasites were counted using the oil 100x magnification under Phase condition. The average number of protruded parasites was determined by counting 60 parasites for each condition for three independent experiments. Data are presented as mean values \pm SD from three independent experiments.

\section{Western blot}

Tachyzoites were prepared as described above and treated for 0,2 , or 5 minutes. The tachyzoites were pelleted from the soup and both fractions were boiled for $5 \mathrm{~min}$, separated by SDS-PAGE, and transferred to polyvinylidene difluoride (PVDF) membranes. Secreted TgMIC2 was detected by incubation of membrane with mouse anti-TgMIC2 antibody (6D10, ascites) and followed by incubation with horseradish-peroxidase (HRP) conjugated goat anti-mouse IgG. The levels of HRP were detected using an enhanced chemiluminescence (ECL) kit (Pierce). SAG1 levels were used to control for the concentration of parasites within the samples using rabbit anti-SAG1 followed by staining with secondary HRP-conjugated goat anti-rabbit IgG antibody and detecting the levels of HRP as described above.

\section{Cryogenic electron tomography and reconstruction}

Glow discharge lacey carbon EM grids were mounted on a manual plunger, loaded with parasite suspension mixed with $10 \mathrm{~nm}$ gold fiducials (EMS), blotted from the back side using Whatman paper 5, and plunged into liquid ethane at near liquid nitrogen temperature. 
The stimulated tachyzoites were imaged using a Talos Arctica electron microscope (Thermo Fisher) equipped with a field emission gun operated at 200kV, a Volta Phase Plate [37], an energy filter (Gatan) operated at zeroloss and a K2 Summit direct electron detector (Gatan). Upon phase plate alignment and conditioning, tilt series of the parasites were recorded at 39,000x at pixel size $3.54 \AA$ Asing Tomo 4 software with bidirectional acquisition schemes, each from $-60^{\circ}$ to $60^{\circ}$ with $2^{\circ}$ or $3^{\circ}$ increment. Target defocus was set to $-1.0 \mu \mathrm{m}$. The $\mathrm{K} 2$ camera was operated in dose fractionation mode recording frames every 0.6s. Every 2 or 3 tilt series, a new spot on the phase plate was selected. The phase shift spans a range from 0.2-0.8 $\pi$. The total dose was limited to 70-90 e/Å2.

The non-stimulated tachyzoites were imaged using a Titan Krios electron microscope (Thermo Fisher) equipped with a field emission gun operated at $300 \mathrm{kV}$, objective aperture, an energy filter (Gatan) operated at zero-loss and a K2 Summit direct electron detector (Gatan). Tilt series of the parasites were recorded at 39,000x at pixel size 3.54 Å. Tilt series were recorded using Tomo 4 software with bidirectional acquisition schemes, each from $-60^{\circ}$ to $60^{\circ}$ with $2^{\circ}$ or $3^{\circ}$ increment. Target defocus was set to -1.0 to $\mu \mathrm{m}$. The total dose was limited to $60-100 \mathrm{e} / \AA \AA 2$. The movie frames were motion-corrected using motionCor2 [38], and the resulting micrographs are compiled into tilt series. Tilt series alignment and reconstruction was performed using IMOD [39, 40]. The reconstructed tomograms are the result of multiple frozen grids that were imaged over several multiple-day sessions.

\section{Mixed-Scale Dense Networks and Iterative Augmentation}

We trained on 20 initial annotated images from 7 different tomograms to build an initial network. We then added an additional 43 annotated images to the training set by allowing the network to extract the major features, and then manually made corrections: the initial predictions were quite accurate, requiring only limited corrections. These corrections, for the most part, were in the form of correcting missing area of AVs, rhoptries and IMTs structures. The network was then trained on the entire, annotated set.

In each case, we trained an MSD network with hyperparameters that are typically used in other studies as well: a width of one, a depth of 100, and dilations uniformly chosen between one and ten, resulting in a network with 49574 learnable parameters. The network input consisted of the 2D cryo-ET slice to be annotated, with four adjacent slices (two above and two below) used as additional input. During training, the manual annotations were used as training target, minimizing the Dice loss. To artificially increase the amount of available training data, the data was augmented using random $90^{\circ}$ rotations and flips. All NN computations were performed using the code accompanying [25] which is GPU-accelerated and available under an open-source license (https://github.com/dmpelt/msdnet). Training was terminated when no significant improvement in the loss was observed, which was typically after a few days.

\section{Meta data extraction from annotated tomograms}

Each of the following quantifications used all tomograms that resolved the feature of interest. Top views were not included in the analysis since the relevant features are not well resolved.

Connected components, surfaces, volumes, and centers of mass were calculated using the image processing toolbox within MATLAB version R2020b. All distances between structures were calculated in three dimensions unless otherwise specified. Average vesicle curvature values shown in figure $4 \mathrm{~B}$ were calculated using the central 11 xy slices $( \pm 7.5 \mathrm{~nm})$ of each vesicle. These measurements were then averaged for each class of vesicle to produce the plots in figure 4B. Vesicle volume measurements were scaled by a factor of 1.33 to account for the missing wedge. This factor was estimated from central slices of IMT-associated AVs that were assumed to be spherical. Incorporation of this factor produces values closer to the true volume of the vesicles than a sum of annotated voxels alone.

\section{References}

1. Battle KE, Lucas TCD, Nguyen M, Howes RE, Nandi AK, Twohig KA, et al. Mapping the Global Endemicity and Clinical Burden of Plasmodium Vivax, 2000-17: a Spatial and Temporal Modelling Study. The Lancet. 2019;394(10195):332-343. doi:10.1016/S0140-6736(19)31096-7/ATTACHMENT/307DF8C0-AF614F1E-B88E-116F1B6176D2/MMC1.PDF. 
2. Furtado JM, Smith JR, Belfort R, Gattey D, Winthrop KL. Toxoplasmosis: A Global Threat. Journal of Global Infectious Diseases. 2011;3(3):281. doi:10.4103/0974-777X.83536.

3. Bigna JJ, Tochie JN, Tounouga DN, Bekolo AO, Ymele NS, Youda EL, et al. Global, Regional, and Country Seroprevalence of Toxoplasma Gondii in Pregnant Women: a Systematic Review, Modelling and Metaanalysis. Scientific Reports. 2020;10(1):1-10. doi:10.1038/s41598-020-69078-9.

4. Morrissette NS, Sibley LD. Cytoskeleton of Apicomplexan Parasites. Microbiology and Molecular Biology Reviews. 2002;66(1):21-38. doi:10.1128/MMBR.66.1.21-38.2002.

5. Dos Santos Pacheco N, Tosetti N, Koreny L, Waller RF, Soldati-Favre D. Evolution, Composition, Assembly, and Function of the Conoid in Apicomplexa. Trends in Parasitology. 2020;36(8):688-704. doi:10.1016/J.PT.2020.05.001.

6. Carruthers V, Boothroyd JC. Pulling Together: an Integrated Model of Toxoplasma Cell Invasion. Current Opinion in Microbiology. 2007;10(1):83-89. doi:10.1016/J.MIB.2006.06.017.

7. Hu K, Roos DS, Murray JM. A Novel Polymer of Tubulin Forms the Conoid of Toxoplasma Gondii. Journal of Cell Biology. 2002;156(6):1039-1050. doi:10.1083/JCB.200112086.

8. Sun SY, Segev-Zarko L, Chen M, Pintilie GD, Schmid MF, Boothroyd JC, et al. Cryo-ET Reveals Two Major Tubulin-Based Cytoskeleton Structures in Toxoplasma Gondii. Proceedings of the National Academy of Sciences: Forthcoming. 2022;doi:10.1101/2021.05.23.445366.

9. Hu K, Johnson J, Florens L, Fraunholz M, Suravajjala S, DiLullo C, et al. Cytoskeletal Components of an Invasion Machine-The Apical Complex of Toxoplasma Gondii. PLOS Pathogens. 2006;2(2):e13. doi:10.1371/JOURNAL.PPAT.0020013.

10. Nichols BA, Chiappino ML. Cytoskeleton of Toxoplasma Gondii. The Journal of Protozoology. 1987;34(2):217-226. doi:10.1111/J.1550-7408.1987.TB03162.X.

11. Dubois DJ, Soldati-Favre D. Biogenesis and Secretion of Micronemes in Toxoplasma Gondii. Cell Microbiology. 2019;21(5). doi:10.1111/CMI.13018.

12. Ben Chaabene R, Lentini G, Soldati-Favre D. Biogenesis and Discharge of the Rhoptries: Key Organelles for Entry and Hijack of Host Cells by the Apicomplexa. Molecular Microbiology. 2021;115(3):453-465. doi:10.1111/MMI.14674.

13. Alexander DL, Mital J, Ward GE, Bradley P, Boothroyd JC. Identification of the Moving Junction Complex of Toxoplasma Gondii: A Collaboration Between Distinct Secretory Organelles. PLoS Pathogens. 2005;1(2):0137-0149. doi:10.1371/JOURNAL.PPAT.0010017.

14. Paredes-Santos TC, de Souza W, Attias M. Dynamics and 3D Organization of Secretory Organelles of Toxoplasma Gondii. Journal of Structural Biology. 2012;177(2):420-430. doi:10.1016/J.JSB.2011.11.028.

15. Aquilini E, Cova MM, Mageswaran SK, Dos Santos Pacheco N, Sparvoli D, Penarete-Vargas DM, et al. An Alveolata Secretory Machinery Adapted to Parasite Host Cell Invasion. Nature Microbiology. 2021;6(4):425-434. doi:10.1038/s41564-020-00854-z.

16. Sheffield HG. Electron Microscope Study of the Proliferative Form of Besnoitia Jellisoni. The Journal of Parasitology. 1966;52(3):583-594. doi:10.2307/3276331.

17. Dubremetz JF, Torpier G. Freeze Fracture Study of the Pellicle of an Eimerian Sporozoite (Protozoa, Coccidia). Journal of Ultrastructure Research. 1978;62(2):94-109. doi:10.1016/S0022-5320(78)90012-6.

18. Porchet-Hennere E, Nicolas G. Are Rhoptries of Coccidia Really Extrusomes? Journal of Ultrastructure Research. 1983;84(2):194-203. doi:10.1016/S0022-5320(83)90130-2.

19. Mageswaran SK, Guérin A, Theveny LM, Chen WD, Martinez M, Lebrun M, et al. In Situ Ultrastructures of Two Evolutionarily Distant Apicomplexan Rhoptry Secretion Systems. Nature Communications. 2021;12(1):1-12. doi:10.1038/s41467-021-25309-9. 
20. Weber M, Wojtynek M, Medalia O. Cellular and Structural Studies of Eukaryotic Cells by Cryo-Electron Tomography. Cells. 2019;8(1):57. doi:10.3390/CELLS8010057.

21. Mondragon R, Frixione E. Ca(2+)-Dependence of Conoid Extrusion in Toxoplasma Gondii Tachyzoites. The Journal of Eukaryotic Microbiology. 1996;43(2):120-127. doi:10.1111/J.1550-7408.1996.TB04491.X.

22. Carruthers VB, Sibley LD. Mobilization of Intracellular Calcium Stimulates Microneme Discharge in Toxoplasma Gondii. Molecular Microbiology. 1999;31(2):421-428. doi:10.1046/J.1365-2958.1999.01174.X.

23. Endo T, Tokuda H, Yagita K, Koyama T. Effects of Extracellular Potassium on Acid Release and Motility Initiation in Toxoplasma Gondii. The Journal of protozoology. 1987;34(3):291-295. doi:10.1111/J.15507408.1987.TB03177.X.

24. Moudy R, Manning TJ, Beckers CJ. The Loss of Cytoplasmic Potassium Upon Host Cell Breakdown Triggers Egress of Toxoplasma Gondii. The Journal of Biological Chemistry. 2001;276(44):41492-41501. doi:10.1074/JBC.M106154200.

25. Pelt DM, Sethian JA. A Mixed-Scale Dense Convolutional Neural Network for Image Analysis. Proceedings of the National Academy of Sciences. 2017;115(2):254-259. doi:10.1073/PNAS.1715832114//DCSUPPLEMENTAL.

26. Tagoe DNA, Drozda AA, Coppens I, Coleman BI, Gubbels MJ. Toxoplasma Ferlin1 is a Versatile and Dynamic Mediator of Microneme Trafficking and Secretion. bioRxiv. 2020; p. 2020.04.27.063628. doi:10.1101/2020.04.27.063628.

27. Farrell A, Thirugnanam S, Lorestani A, Dvorin JD, Eidell KP, Ferguson DJP, et al. A DOC2 Protein Identified by Mutational Profiling is Essential for Apicomplexan Parasite Exocytosis. Science. 2012;335(6065):218-221. doi:10.1126/SCIENCE.1210829/SUPPL_FILE/1215141.FARRELL.SOM.PDF.

28. Friedrich R, Yeheskel A, Ashery U. DOC2B, C2 Domains, and Calcium: A Tale of Intricate Interactions. Molecular Neurobiology. 2010;41(1):42-51. doi:10.1007/\$12035-009-8094-8.

29. Tagoe DNA, Drozda AA, Falco JA, Bechtel TJ, Weerapana E, Gubbels MJ. Ferlins and TgDOC2 in Toxoplasma Microneme, Rhoptry and Dense Granule Secretion. Life. 2021;11(3). doi:10.3390/LIFE11030217.

30. Monteiro VG, De Melo EJT, Attias M, De Souza W. Morphological Changes During Conoid Extrusion in Toxoplasma Gondii Tachyzoites Treated With Calcium lonophore. Journal of Structural Biology. 2001;136(3):181-189. doi:10.1006/JSBI.2002.4444.

31. Burrell A, Marugan-Hernandez V, Moreira-Leite F, Ferguson D, Tomley F, Vaughan S. Cellular Electron Tomography of the Apical Complex in the Apicomplexan Parasite Eimeria Tenella Shows a Highly Organised Gateway For Regulated Secretion. bioRxiv. 2021;doi:10.1101/2021.06.17.448283.

32. Coleman BI, Saha S, Sato S, Engelberg K, Ferguson DJP, Coppens I, et al. A Member of the Ferlin Calcium Sensor Family Is Essential for Toxoplasma Gondii Rhoptry Secretion. mBio. 2018;9(5). doi:10.1128/MBIO.01510-18.

33. Koshy AA, Dietrich HK, Christian DA, Melehani JH, Shastri AJ, Hunter CA, et al. Toxoplasma Co-Opts Host Cells It Does Not Invade. PLOS Pathogens. 2012;8(7):e1002825. doi:10.1371/JOURNAL.PPAT.1002825.

34. Dubremetz JF. Rhoptries are Major Players in Toxoplasma Gondii Invasion and Host Cell Interaction. Cellular Microbiology. 2007;9(4):841-848. doi:10.1111/J.1462-5822.2007.00909.X.

35. Wu GH, Mitchell PG, Galaz-Montoya JG, Hecksel CW, Sontag EM, Gangadharan V, et al. Multiscale 3D Cryo-Correlative Microscopy for Vitrified Cells. Structure. 2020;28(11):1231-1237. doi:10.1016/J.STR.2020.07.017.

36. Dahlberg PD, Moerner WE. Cryogenic Super-Resolution Fluorescence and Electron Microscopy Correlated at the Nanoscale. Annual Review of Physical Chemistry. 2021;72:253-278. doi:10.1146/ANNUREVPHYSCHEM-090319-051546. 
37. Danev R, Buijsse B, Khoshouei M, Plitzko JM, Baumeister W. Volta Potential Phase Plate for In-Focus Phase Contrast Transmission Electron Microscopy. Proceedings of the National Academy of Sciences. 2014;111(44):15635-15640. doi:10.1073/PNAS.1418377111/-/DCSUPPLEMENTAL.

38. Zheng SQ, Palovcak E, Armache JP, Verba KA, Cheng Y, Agard DA. MotionCor2: Anisotropic Correction of Beam-Induced Motion for Improved Cryo-Electron Microscopy. Nature Methods. 2017;14(4):331-332. doi:10.1038/nmeth.4193.

39. Mastronarde DN. Dual-Axis Tomography: An Approach With Alignment Methods That Preserve Resolution. Journal of Structural Biology. 1997;120(3):343-352. doi:10.1006/JSBI.1997.3919.

40. Kremer JR, Mastronarde DN, McIntosh JR. Computer Visualization of Three-Dimensional Image Data Using IMOD. Journal of Structural Biology. 1996;116(1):71-76. doi:10.1006/JSBI.1996.0013.

\section{Conflict of interest}

The authors declare no competing interests.

\section{Data availability}

The tomograms of the Toxoplasma apical end are deposited to EMDB with the accession code D_1000262131 for the tomogram of the stimulated tachyzoite, and D_1000262118 for the tomogram of the non-stimulated tachyzoite.

\section{Acknowledgments}

We thank all members of our respective research groups for always helpful input and especially Melanie Espiritu for growth of fibroblasts and Dr. Michael F. Schmid for particularly insightful comments during analysis of the data. We thank the support of Chan Zuckerberg Biohub Intercampus Team Award for supporting this research. Other support includes NIH grants: S100D021600, P41GM103832, P01GM121203, and the Center for Advanced Mathematics for Energy Research Application (CAMERA), jointly funded by The Office of Advanced Scientific Research (ASCR) and the Office of Basic Energy Sciences (BES) within the DOE's Office of Science at the Lawrence Berkeley National Laboratory, under contract number DE0AC02-5CH11231. L.S-Z. was supported in part by BARD, the United States - Israel Binational Agricultural Research and Development Fund, Vaadia-BARD Postdoctoral Fellowship Award No. FI-582-2018, the Stanford Maternal and Child Health Research Institute, and Stanford School of Medicine Dean's Postdoctoral Fellowship. P.D.D was supported in part by the National Institute of General Medical Sciences grant R35-GM118067. D.M.P. was supported in part by The Netherlands Organization for Scientific Research (NWO), project number 016.Veni.192.235.

\section{Author contributions}

Conceptualization: L.S-Z., P.D.D., D.M.P., S.Y.S., J.A.S., W.C., J.C.B.; formal analysis, investigation, and visualization, L.S-Z., P.D.D., S.Y.S., D.M.P.; resources, L.S-Z., P.D.D., S.Y.S., D.M.P.; methodology, L.S-Z., P.D.D., D.M.P., S.Y.S.; software, and data curation, D.M.P., P.D.D.; writing - Original Draft, L.S-Z.; writing - Review \& Editing, L.S-Z., P.D.D., D.M.P., S.Y.S., J.A.S., W.C., J.C.B.; funding acquisition and supervision, J.C.B., W.C., J.A.S. 


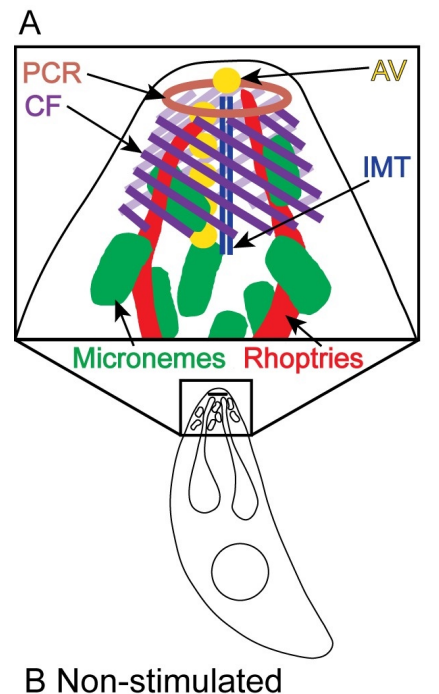

\section{Non-stimulated}
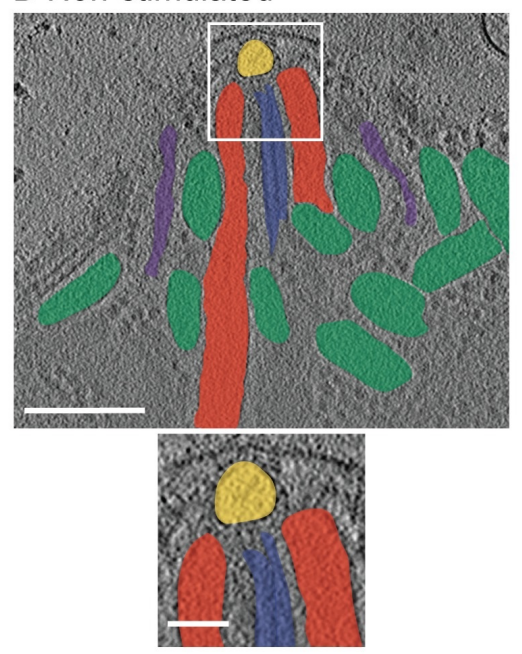

\section{E Stimulated}
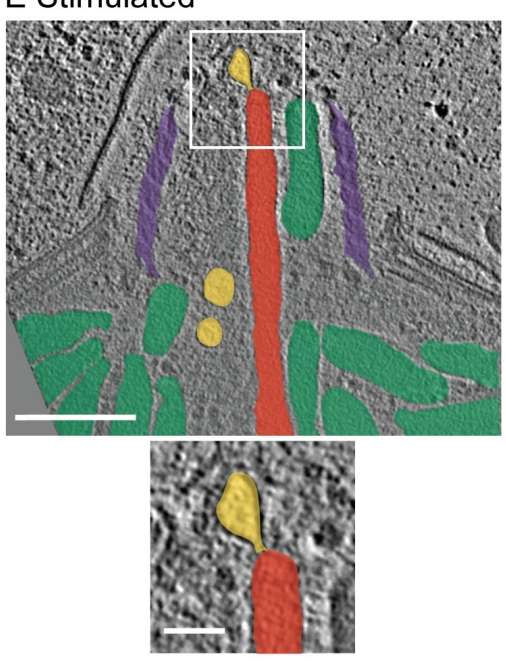
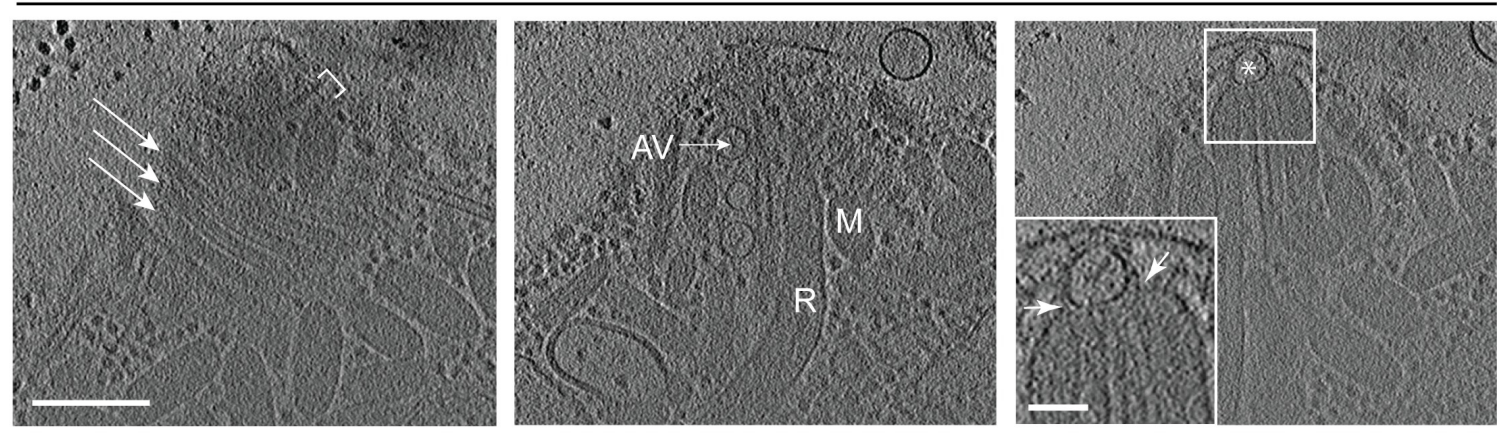

C Stimulated
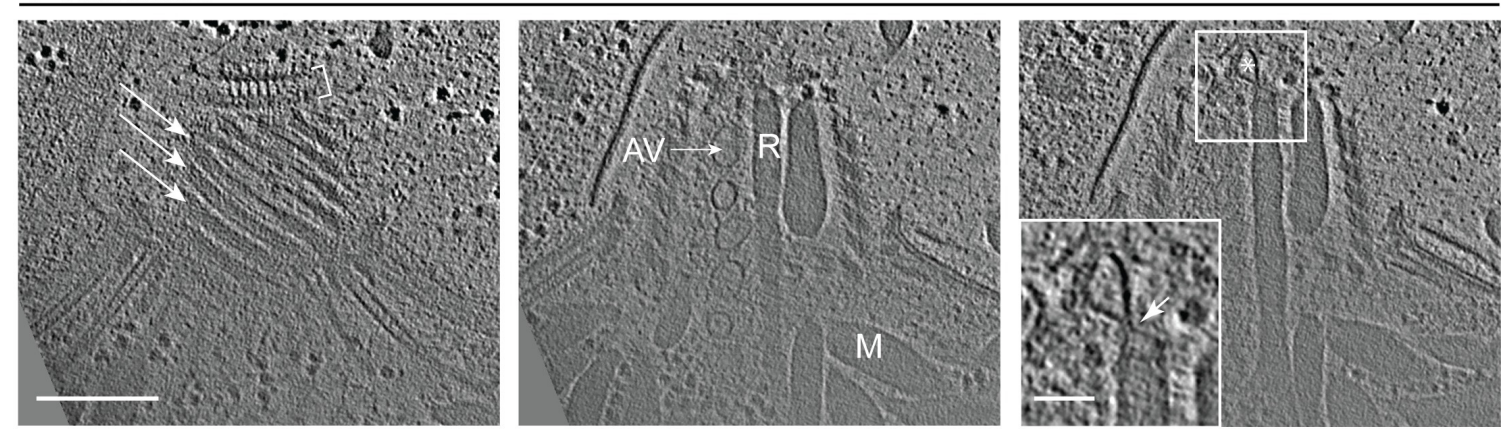

Fig 1. Cryo-ET reveals the organization of subcellular organelles in the apical complex upon ionophorestimulation. (A) Cartoon of the Toxoplasma tachyzoite cell as well as an enlarged cartoon of the apical complex showing key subcellular structures: preconoidal rings (PCR; brown), conoid fibrils (CF; purple), intra-conoidal microtubules (IMT; blue), micronemes (green), rhoptries (red) and apical vesicles (AV; yellow). (B) Tomographic slices of a representative apical complex of non-stimulated tachyzoite showing the preconoidal rings (brackets), conoid fibrils (arrows), micronemes (M), rhoptries (R), IMT-associated AVs (AV), and the most anterior AV (asterisk). Scale bar, $200 \mathrm{~nm}$. Inset is a zoomed-in view of the square showing the association (arrowheads) of the rhoptries with an AV. Scale bar, $50 \mathrm{~nm}$. (C) as for (B) except the images are of a tachyzoite stimulated with ionophore, and the zoomed-in view of the square showing the interaction (arrowhead) of a rhoptry tip with an AV. (D) and (E) are the slices shown in the far right of (B) and (C), respectively, with structures highlighted in colors as (A), and a zoomed in view of the square. Movies with the complete tomograms are available in S1 Movie(nonstimulated) and S2 Movie(ionophore-stimulated). 
bioRxiv preprint doi: https://doi.org/10.1101/2022.01.12.476068; this version posted January 12, 2022. The copyright holder for this preprint (which was not certified by peer review) is the author/funder, who has granted bioRxiv a license to display the preprint in perpetuity. It is made available under aCC-BY-NC-ND 4.0 International license.

A

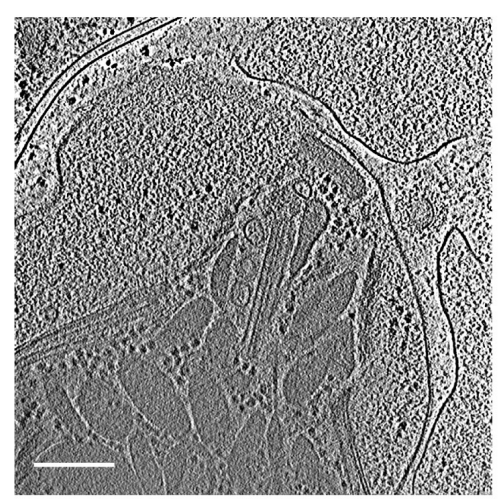

$\underline{B}$

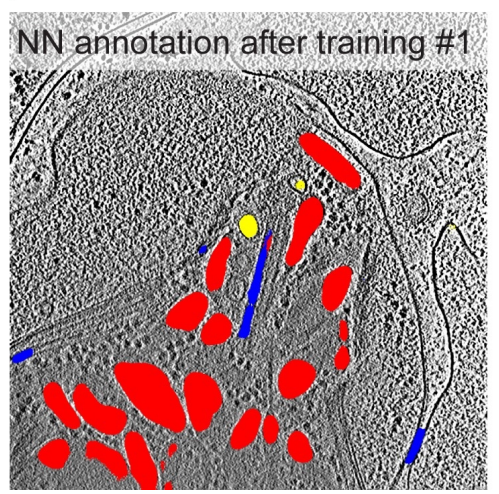

C

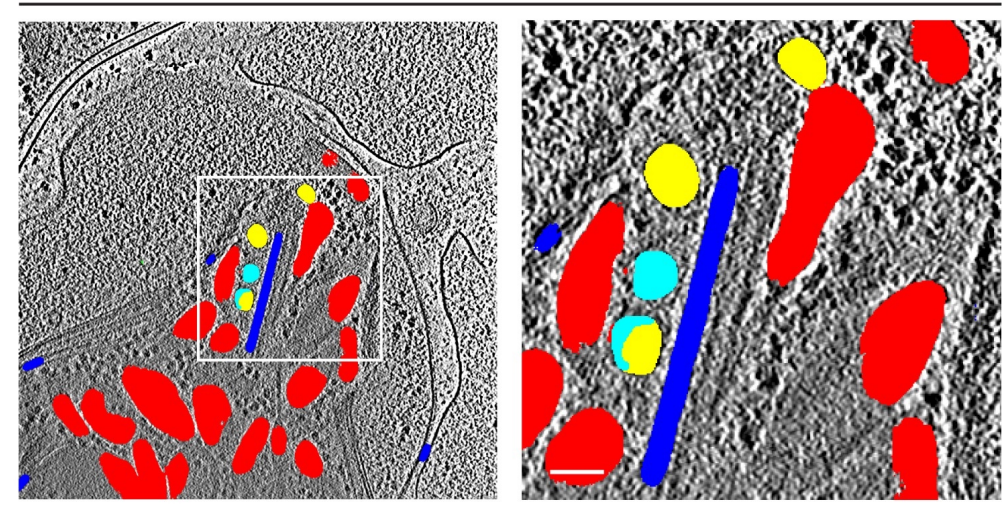

NN annotation after training \#2

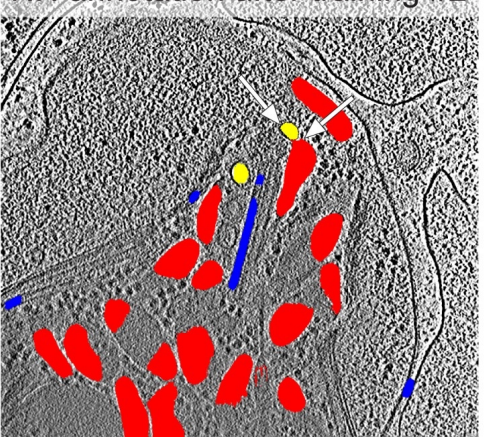

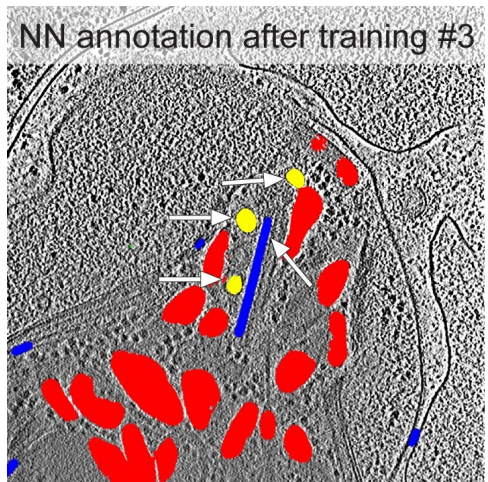

Fig 2. Annotation of distinct elements in the apical complex of Toxoplasma tachyzoites using a mixed-scale dense convolutional neural network. (A) A representative slice from a tomogram that was not used in the training data and that is shown annotated in (B) and (C). Scale bar, $200 \mathrm{~nm}$. (B) Annotation produced by the neural network after different iterative rounds of training (see results section for details) overlaid on the tomographic slice from (A) showing the AVs (yellow), rhoptries and micronemes (red), and microtubules (blue). Note the small but marked improvement in the annotation accuracy of the AVs, rhoptry, and IMT with each additional training as pointed by the arrows. (C) Manual correction (cyan) of the NN annotation after training 3. A zoomed-in view of the square in the left panel is shown in the right panel. Scale bar, $50 \mathrm{~nm}$. 
A

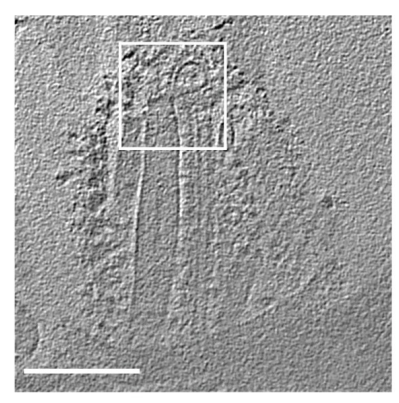

C

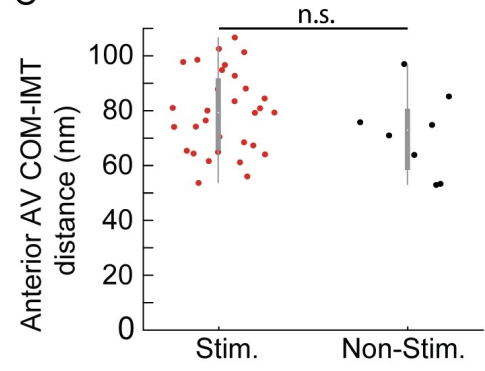

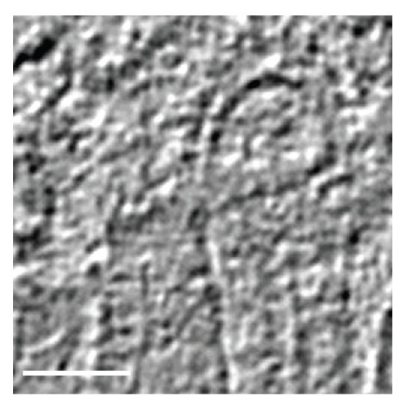

D

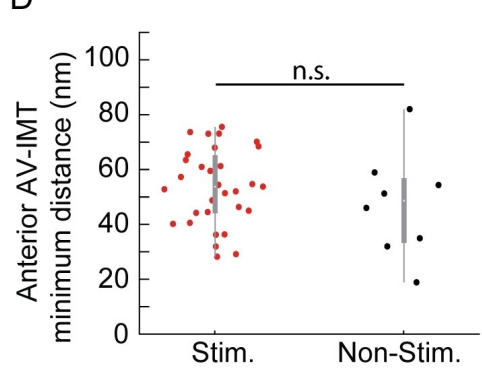

B
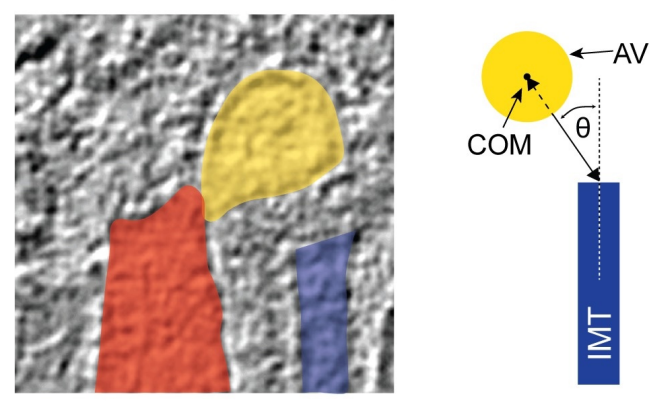

$\mathrm{E}$

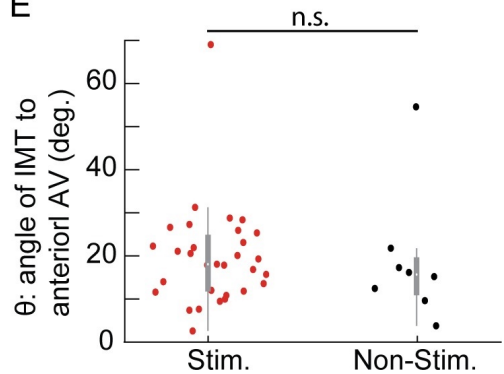

Fig 3. The positioning of the anterior AV relative to the IMT does not change upon ionophore-stimulation. (A) Left panel: a tomographic slice of the AC of an ionophore-stimulated tachyzoite. Scale bar, $200 \mathrm{~nm}$. Middle panel: a zoomed-in view of the square in the left panel. Scale bar, $50 \mathrm{~nm}$. Right panel: as for the middle panel image but highlighting in color the tip of the rhoptry (red), anterior AV (yellow), and IMT (blue). (B) A schematic showing the parameters used to assess the relative positions of the IMT (blue) and anterior AV (yellow). (C) The distance from the anterior AV's center-of-mass (COM) to the tip of the IMT in stimulated (Stim.; mean \pm SD $=79.3 \pm 14.7$ $\mathrm{nm}$, median=79.3 nm, N=31) and non-stimulated (Non-Stim.; mean $\pm \mathrm{SD}=71.8 \pm 17.1 \mathrm{~nm}$ median=72.9 $\mathrm{nm}, \mathrm{N}=8$ ) tachyzoites. Each dot is the result for one tomogram. (D) As for (C) except the distance from the IMT tip to the closest part of the anterior AV's membrane is shown. Stimulated; mean $\pm S D=53.3 \pm 13.8 \mathrm{~nm}$ median $=53.5 \mathrm{~nm}$, $\mathrm{N}=31$ and non-stimulated; mean $\pm \mathrm{SD}=47.1 \pm 19.2 \mathrm{~nm}$ median $=48.4 \mathrm{~nm}, \mathrm{~N}=8$. (E) The angle ([]) subtended by the long axis of the closest IMT and a line connecting the COM of the AV to the IMT tip. Stimulated; mean \pm SD $=19.6 \pm 11.7^{\circ}$, median $=18^{\circ}, \mathrm{N}=31$ and non-stimulated; mean $\pm \mathrm{SD}=18.8 \pm 15.4^{\circ}$, median $=15.6^{\circ}, \mathrm{N}=8$. There was no statistically significant difference ("n.s.") between the stimulated and non-stimulated parasites for any of these three comparisons. 


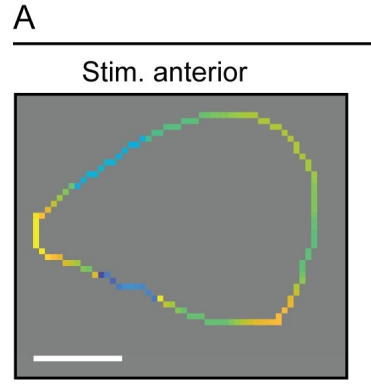

B

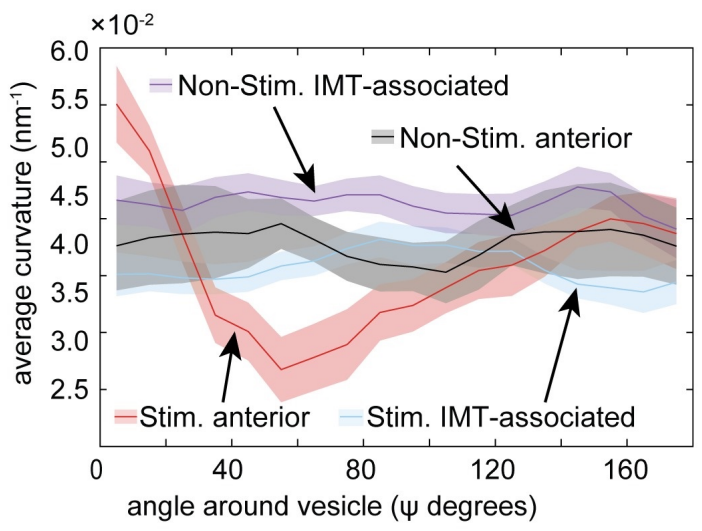

$\mathrm{D}$

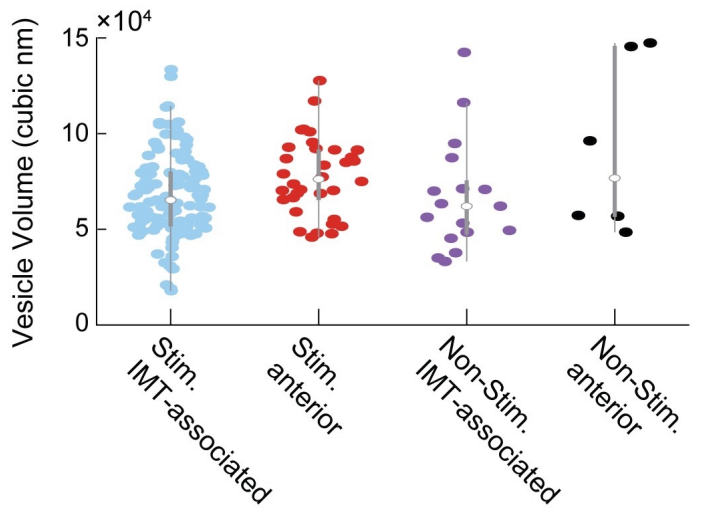

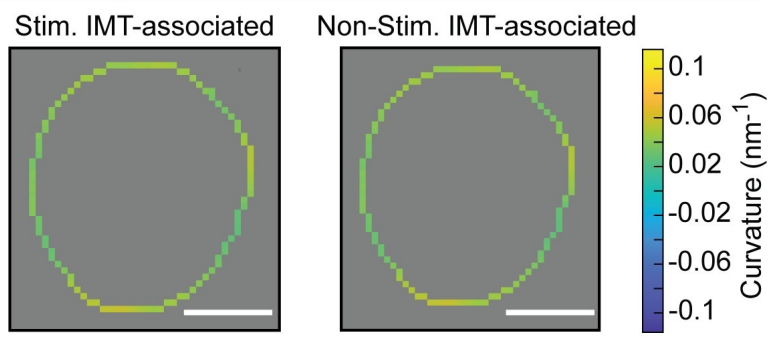

C

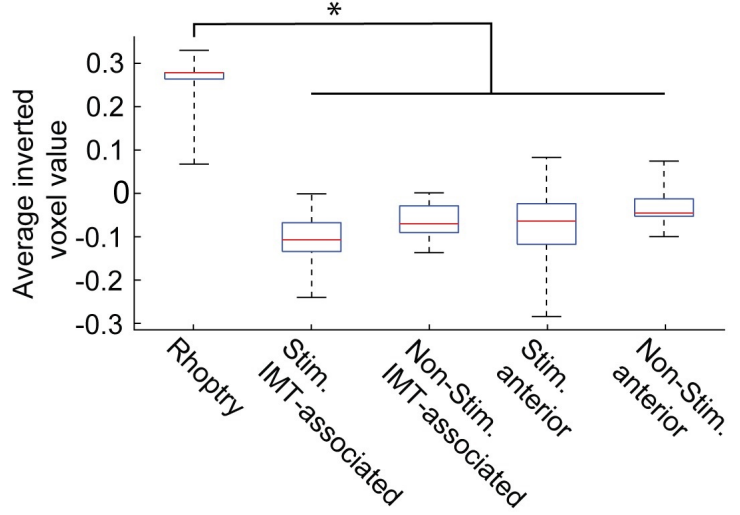

Fig 4. Ionophore-stimulation results in a distortion of the anterior AV's shape but not its density relative to the other AVs. (A) The curvature of representative anterior and IMT-associated AVs. The curvature around the vesicles was measured for each pixel on the periphery of the vesicle in xy slices. See Materials and methods for more details. Vesicles were aligned by their shortest distance between each vesicle's COM and either the rhoptry tip or the IMT, for anterior and IMT-associated vesicles, respectively (see details in S5 Fig). The heatmap reflects the positive and negative curvature as "warmer" and "cooler" colors, respectively. Scale bar $20 \mathrm{~nm}$. The three-dimensional (3-D) annotation of the AVs are presented in S1 Movie (non-stimulated) and S2 Movie and S4 Movie (stimulated). Note that the tear drop shape was also apparent in 3-D. (B) The averaged curvature for all AVs in stimulated (Stim.) and non-stimulated (Non-Stim.) tachyzoites, measured every 10 degrees as in (A). (C) The density of the rhoptries (mean \pm SD $=-0.259 \pm 0.054, N=35$ ), and anterior and IMT-associated AVs under stimulated (Stim.; anterior mean \pm SD $=0.082 \pm 0.083, \mathrm{~N}=34$, and IMT associated mean \pm SD $=0.103 \pm 0.048$, $\mathrm{N}=119$ ) and non-stimulated (Non-Stim.; anterior mean $\pm \mathrm{SD}=0.03 \pm 0.058, \mathrm{~N}=6$, and IMT associated mean $\pm \mathrm{SD}$ $=0.656 \pm 0.042, N=17)$ conditions. The only statistically significant difference $(p<0.05)$ was between the rhoptry and the AVs in each of the four conditions. (D) The volumes of the anterior and IMT-associated AVs under stimulated (Stim.; anterior mean \pm SD $=7.7 \pm 2$ X104 nm3, N=34, and IMT-associated mean \pm SD $=6.8 \pm 2.2$ X104 nm3, $\mathrm{N}=119$ ) and non-stimulated (Non-Stim.; anterior mean $\pm \mathrm{SD}=9.2 \pm 4.5 \mathrm{X} 104 \mathrm{~nm} 3, \mathrm{~N}=6$, and IMT-associated mean $\pm \mathrm{SD}=6.7 \pm 2.9 \times 104 \mathrm{~nm} 3, \mathrm{~N}=17$ ) conditions. None of the pair-wise comparisons showed a statistically significant difference. 
A

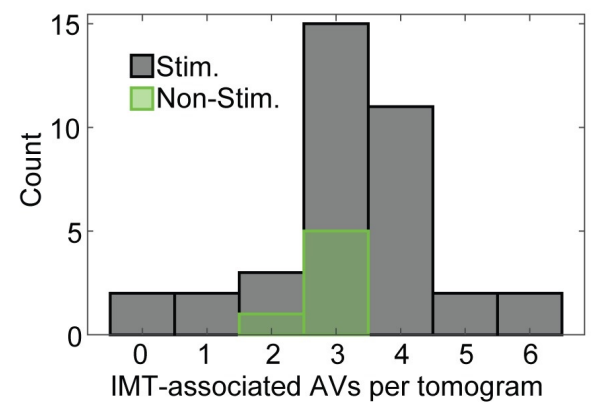

B

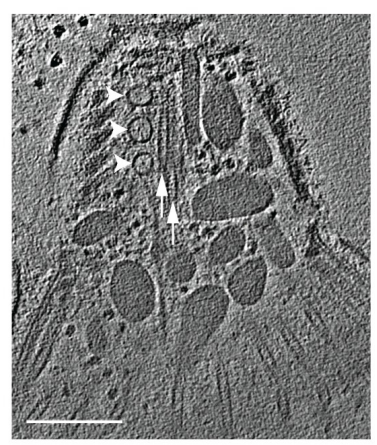

C

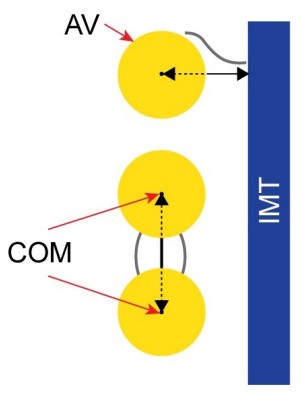

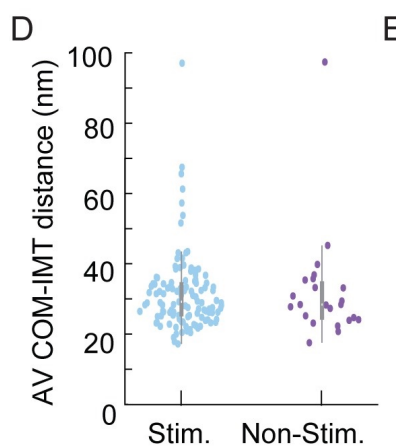

$\mathrm{E}$

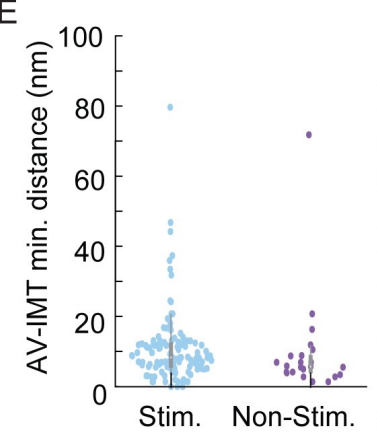

$\mathrm{H}$

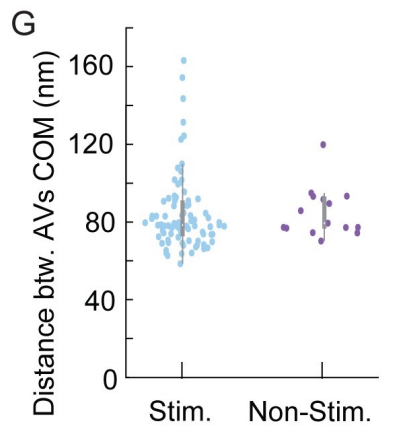

F

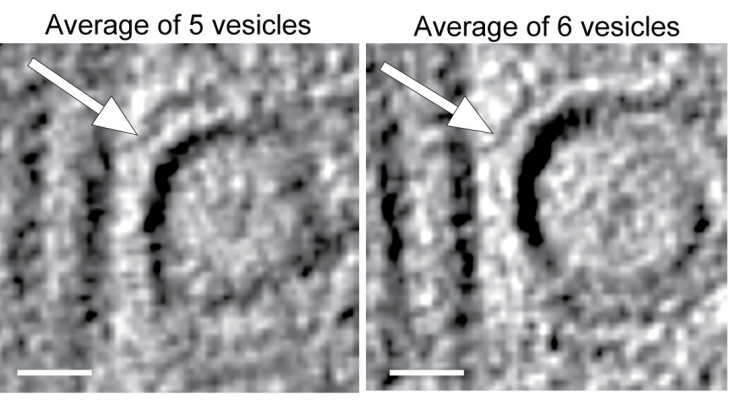

।

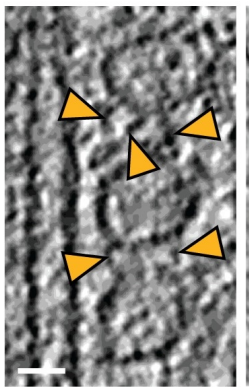

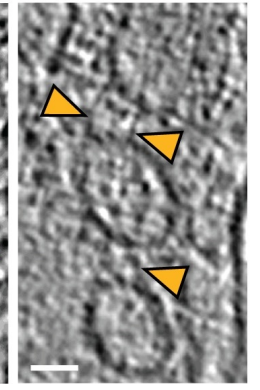

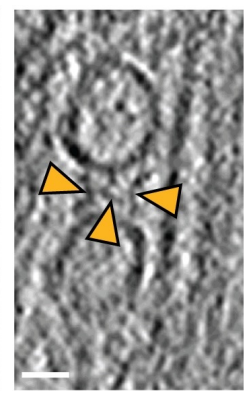

Fig 5. The AVs interact with the IMTs and with each other. (A) The number of IMT-associated AVs in the AC of ionophore-stimulated (Stim.) and non-stimulated (Non-Stim.) tachyzoites. (B) A tomographic slice of the AC of a stimulated tachyzoite showing three AVs (arrowheads) associated with the IMTs (arrows). Scale bar, $200 \mathrm{~nm}$. (C) A schematic showing the parameters used to assess the distance between the AVs (yellow) and between each AV and the IMT (blue). Measurements began at either the AVs' COM (red arrows; middle and bottom AVs) or their delimiting membrane (red arrow; top AV). (D) The distance from each AV's COM to the IMT in stimulated (Stim.; mean $\pm \mathrm{SD}=32.1 \pm 15.6 \mathrm{~nm}$ median $=28.4 \mathrm{~nm}, \mathrm{~N}=23$ ) and non-stimulated (Non-Stim.; mean $\pm \mathrm{SD}=32.1 \pm 15.6$ $\mathrm{nm}$ median=28.4 nm, N=23) tachyzoites. Each dot is the result for one AV. (E) As for (D) except the distance from the AV membrane to the IMT is plotted. (F) Subtomogram averaging of all IMT-associated AVs from two separate tomograms of stimulated tachyzoites. AVs were aligned by the minimum distance between the AVs and the IMT. This averaging reveals an apparent density between the two structures (arrows). Scale bar, $20 \mathrm{~nm}$. (G) The distance between neighboring AV's COM in stimulated (Stim.; mean $\pm S D=84.1 \pm 19.3 \mathrm{~nm}$ median=78.4 $\mathrm{nm}, \mathrm{N}=84$ ) and non-stimulated (Non-Stim.; mean $\pm \mathrm{SD}=85 \pm 12.6 \mathrm{~nm}$ median=79.4 $\mathrm{nm}, \mathrm{N}=15$ ) tachyzoites. (H) As for (G) except the distance between neighboring AV's delimiting membrane is plotted. Stimulated, mean \pm SD $=30.6 \pm 18.5 \mathrm{~nm}$ median $=23.6 \mathrm{~nm}, \mathrm{~N}=84$ and non-stimulated, mean $\pm \mathrm{SD}=34.2 \pm 12.7 \mathrm{~nm}$ median=31.2 nm, N=15 (I) Tomographic slices from 3 ionophore-stimulated tachyzoites showing a density (arrowheads) reproducibly seen between the IMT-associated AVs. Scale bar, $20 \mathrm{~nm}$. No statistically significant difference was seen for any of the pairwise comparisons in the five datasets shown (parts (A), (D), (E), (G) and (H). 